\title{
Immunohistochemical Detection of Tyrosine Hydroxylase and Concentrations of Monoamines in the Substantia Nigra and Hypothalamus of Hereditary Microphthalmic Rats
}

\author{
Su Hyeun SHIM1, 3), Sung J un KIM3), Kunio SUGAHARA2), \\ Shoei SUGITA2), and Hideyuki TANAKA ${ }^{1)}$ \\ ${ }^{1)}$ Departments of Applied Biological Chemistry and ${ }^{2)}$ Animal Science, Faculty of Agriculture, Utsunomiya \\ University, 350 Mine, Utsunomiya 321-8505, Japan, and ${ }^{3)}$ Department of Genetic Engineering, \\ Chosun University, Kwangju 501-759, Korea
}

\begin{abstract}
We compared tyrosine hydroxylase immunoreactivity in the substantia nigra and hypothalamus of hereditary microphthalmic rats with that of normal rats. A considerable number of neuronal cell bodies expressing tyrosine hydroxylase were present in the substantia nigra of the microphthalmic mutant as well as normal rats. Neuronal cells positive for tyrosine hydroxylase in the hypothalamus were fewer than in the substantia nigra in both rats. The concentrations of monoamines (dopamine, noradrenaline, adrenaline, and serotonin) in the substantia nigra and hypothalamus in the microphthalmic mutant were approximately the same as those of normal rats, although the diurnal fluctuation of a few monoamines was observed in normal rats. These results suggest that the metabolic aspects of catecholamine in the substantia nigra and hypothalamus of the microphthalmic mutant rat do not markedly differ from those of normal rats.
\end{abstract}

Key words: catecholamines, microphthalmic rat, tyrosine hydroxylase immunoreactivity

The brains of mutants with anophthalmia or microphthalmia never receive visual input because of the absence of the optic nerve, optic chiasm and optic tract during the morphogenetic period of ontogeny $[2-4,9]$. The congenital absence of visual input to the suprachiasmatic nuclei $(\mathrm{SCN})$ of the hypothalamus might affect the development of circadian rhythms of biochemical and physiological phenomena, behavior, metabolism and neuroendocrine functioning. Such hereditarily blind animals may be useful as a tool for the study of chronobiology to elucidate the interaction be- tween the biological clock(s) and environmental cues on behavioral or metabolic rhythms.

The hereditary microphthalmic rat completely lacks the optic nerve and has been bred in our laboratory [10, $11,13]$. We have previously reported unusual patterns for the circadian rhythms in locomotor activity and metabolic activities such as ornithine decarboxylase and serotonin $\mathrm{N}$-acetyltransferase in blind mutant rats under ad lib feeding conditions [10, 11]. It has also been reported that retino-recipient nuclei such as the lateral geniculate nucleus and superior colliculus of the he-

(Received 18 January 2001 / Accepted 15 May 2001)

Address corresponding: H. Tanaka, Department of Applied Biological Chemistry, Faculty of Agriculture, Utsunomiya University, 350 Mine, Utsunomiya 321-8505, Japan 
reditary microphthalmic rat, the retina of which is seen as a cyst consisting of the pigment layer [6], are 60 to $70 \%$ of the volume of normal rats [15]. Furthermore, in this mutant, the volume of SCN was deduced to be about $70 \%$ of the normal one, and the numbers of vasoactive intestinal polypeptide (VIP) immunoreactive neurons in SCN were significantly decreased [15]. However, the relation of monoaminergic neurons and biogenic amines, catecholamines and indoleamines, which play various crucial roles in the transmission of physiological and periodic information, has not been investigated in the brains of this mutant rat. Dopamine is highly concentrated in the substantia nigra but elsewhere may be rapidly converted by $\beta$-hydroxylation to noradrenaline. In the present study, to clarify whether the catecholaminergic neurons of the microphthalmic rat are abnormal or not, we conducted an immunohistochemical investigation of the catecholamine synthesizing enzyme, tyrosine hydroxylase, in the substantia nigra and hypothalamus of the mutant rat brain. In addition, we investigated the distribution of monoamines (dopamine, noradrenaline, adrenaline and serotonin) in the brain sites of this mutant.

The microphthalmic Donryu rat has been produced through brother-sister mating in our laboratory [11] and the male mutant rats, weighing about $240 \mathrm{~g}$, used in the present experiments were the fourth and ninth generations. The care and treatment of the experimental animals conformed to the Utsunomiya University guidelines of ethical treatment of laboratory animals. Normal male Donryu rats were obtained from a local breeder (SEASCO, Saitama, Japan) as the control. Food and water were supplied ad libitum. All animals were adapted for at least three weeks to an environmental room equipped with a programmed $12 \mathrm{hr}$ dark-12 hr light cycle (light on 5:00 and off 17:00) at a temperature of $22 \pm 2{ }^{\circ} \mathrm{C}$. Illumination was supplied with fluorescent tubes and the light intensities at the cage level were about 45 to $68 \mathrm{~lx}$. The animals were kept in individual cages and fed on a pellet diet containing $20 \%$ casein (Toyo Sangyo, Toyama, Japan).

All animals were deeply anesthetized with sodium pentobarbital $(50 \mathrm{mg} / \mathrm{kg}$ ) and perfused transcardially with $200 \mathrm{~m} l$ of Ringer solution followed by $500 \mathrm{~m} l$ of $1 \%$ paraformaldehyde and $0.1 \mathrm{M} \mathrm{Na-phosphate} \mathrm{buffer}$ $(\mathrm{pH}$ 7.4). The brain was removed and stored in the same fixative at $4{ }^{\circ} \mathrm{C}$ until use. For the immunohis- tochemical analysis, the brain was immersed in $10 \%$ sucrose in the same buffer and cut into serial transverse frozen sections at $40 \mu \mathrm{m}$ using a cryostat [15]. For detection of tyrosine hydroxylase, the brain sections were preincubated for $30 \mathrm{~min}$ at room temperature in $1 \%$ bovine serum albumin (BSA) and $0.2 \%$ Triton X100 in $0.1 \mathrm{M}$ phosphate buffered saline (PBS). After being rinsed two times in $0.5 \%$ BSA for $10-15$ minutes, the sections were incubated with anti-tyrosine hydroxylase (1:25000 in $0.1 \mathrm{M}$ PBS and $0.5 \%$ BSA) overnight in a moist chamber, and then with biotinylated anti-rabbit IgG (Vector Labs, CA, USA; 1:200 in 0.1 $\mathrm{M}$ PBS and $0.5 \% \mathrm{BSA}$ ) for $1 \mathrm{hr}$ at room temperature. The antiserum for tyrosine hydroxylase was provided by Tong-Hyub, Joh (Medical college of Cornell University). After washing with $0.1 \mathrm{M} \mathrm{PBS}$, the sections were incubated with avidin-biotin peroxidase complex (ABC) reagent (Vector Labs, CA, USA) for the immunohistochemical detection of tyrosine hydroxylase.

Determination of brain monoamines was performed, using high performance liquid chromatography (HPLC) followed by electrochemical detection [5]. Two groups of 7 rats each were sacrificed by decapitation at each of 2 time periods selected $(11: 00,23: 00)$ and their brains were frozen quickly on dry ice and stored at $-80^{\circ} \mathrm{C}$ until analysis. After the substantia nigra and hypothalamus were dissected, they were put into a Potter-Elvehjem type micro glass homogenizer and weighed. Substantia nigra $(8-12 \mathrm{mg})$ and hypothalamus $(10-15 \mathrm{mg})$ were homogenized in $200 \mu \mathrm{l}$ of cold $0.05 \mathrm{M}$ perchloric acid $\left(\mathrm{HClO}_{4}\right)$ solution. The tissue homogenate was centrifuged at $5,700 \times \mathrm{g}$ for $5 \mathrm{~min}$ at $4^{\circ} \mathrm{C}$, and the supernatant was then placed in a centrifuge-filtration unit (Millipore, Ultra Free C3-GV) and recentrifuged. The filtrate of 30 $\mu l$ was injected into the HPLC equipment with an Eicompak CA-5 ODS $(4.6 \times 150 \mathrm{~mm})$ column and a electrochemical detector (ECD-100, Eicom Co., Tokyo, Japan). The mobile phase was $0.1 \mathrm{M}$ sodium phosphate buffer, $1.8 \mathrm{mM}$ sodium octyl sulfate, $0.1 \mathrm{mM}$ EDTA2Na and $50 \mathrm{~g} / \mathrm{l}$ methanol, at $\mathrm{pH}$ 6.0. The flow rate was $1 \mathrm{~m} / /$ min using a pump (EP-60, Eicom Co., Tokyo, Japan). Noradrenaline was oxidized with an applied potential of $550 \mathrm{mV}$ against a platinum-silver chloride reference cell. Chromatograms were monitored, recorded and analyzed with a model 807-IT integrator (Nihon Bunko, Tokyo, Japan). Peaks were measured by area and compared with daily calibrations. Samples were analyzed on the 

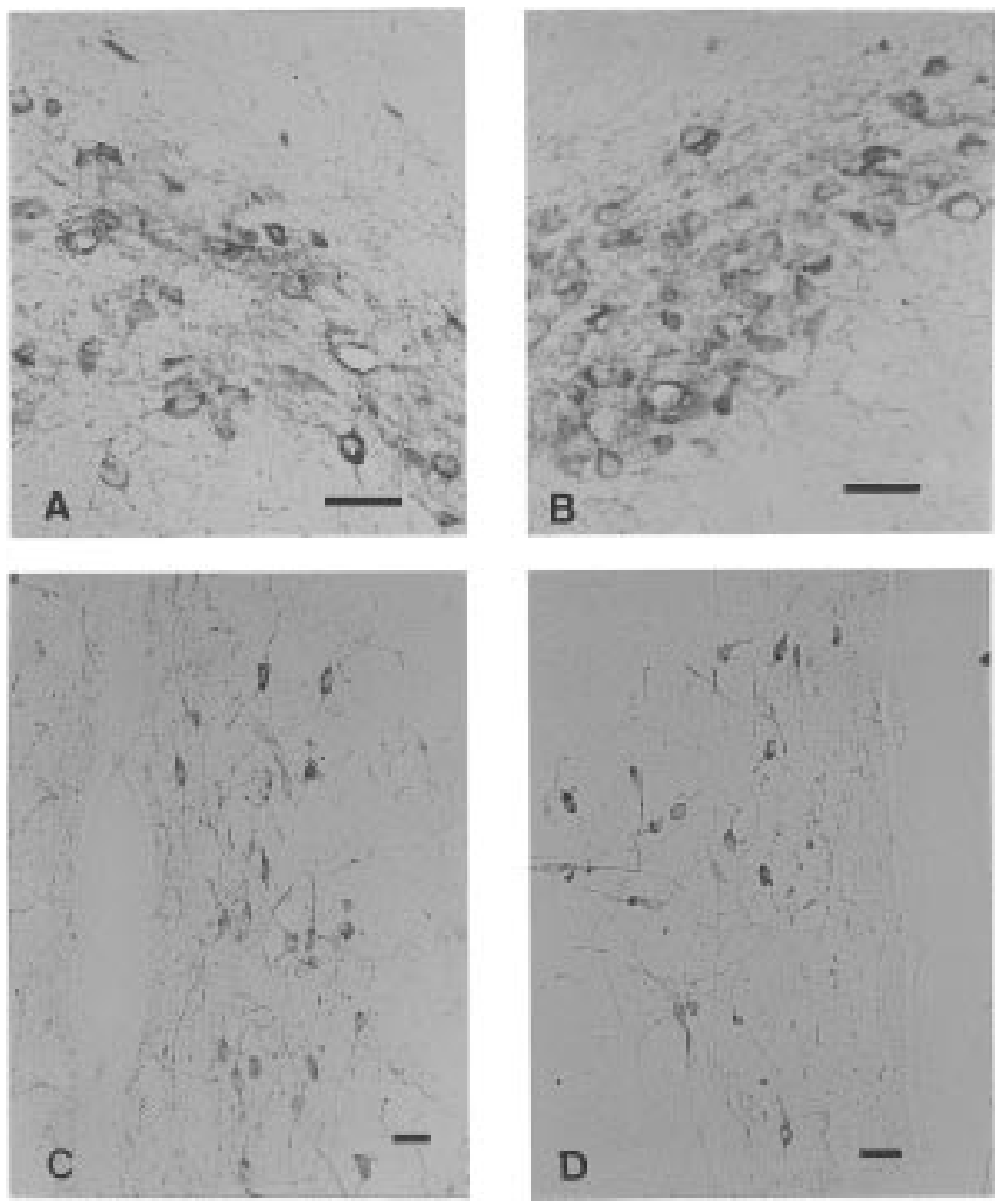

Fig. 1. Photomicrographs of the tyrosine hydroxylase $(\mathrm{TH})$ immunoreactivity (THim) in the substantia nigra (A and B) and hypothalamus (C and D) of normal (A and C) and hereditary microphthalmic (B and D) rats. TH-im is seen in the microphthalmic rat as well as the normal one. Note many TH positive neurons in the substantia nigra of the normal (A) and the microphthalmic (B) rat. Considerable number of $\mathrm{TH}$ positive neurons and fibers are seen in the periventricular hypothalamic area of both rats $(C$ and $D)$. Bars $=25 \mu \mathrm{m}$.

same day of the preparation. The results were analysed by Student's $t$ test or by an analysis of variance. Tukey's test for multiple comparisons was also used to evaluate the statistical significance of the treatment difference. Probability levels less than 0.05 were considered to be significant.

Immunohistochemical analyses of the substantia nigra and hypothalamus were performed on normal Donryu and hereditary microphthalmic rats. The specific staining revealed immunoreactivity for tyrosine hydroxylase in neuron bodies of the substantia nigra and hypothalamus. In the hereditary microphthalmic rats used in this study, the number of neuronal cells expressing tyrosine hydroxylase immunoreactivity in the substantia nigra was similar to that of normal rats (Fig. $1 \mathrm{~A}$ and 1B). Tyrosine hydroxylase immunoreactivity in the hypothalamus of normal and hereditary microphthalmic rats were mainly expressed in the periventriculus tertius (Fig. 1C and 1D), and the number of neuronal cells expressing tyrosine hydroxylase immunoreactivity was similar in both the normal and microphthalmic rats. Immunoreactive substances were found in the soma of the neurons and not in the nucleus of the neurons both in the substantia nigra and hypo- 

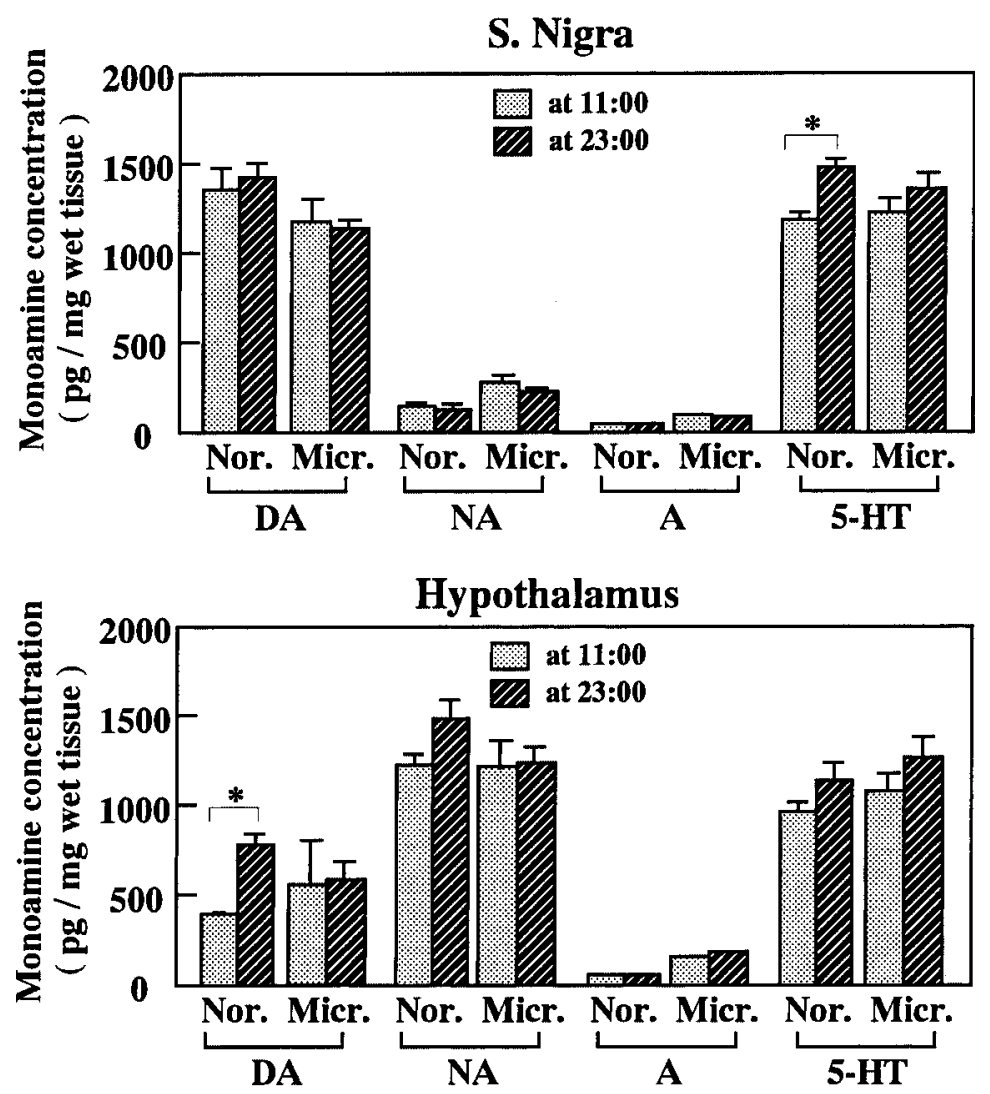

Fig. 2. Diurnal levels of monoamines in the substantia nigra and hypothalamus of normal and hereditary microphthalmic rats. The results are shown as means \pm standard errors $(n=7)$. Asterisks denote significant differences $(\mathrm{p}<0.05)$. There are no significant differences of each monoamine level between normal and microphthalmic rats. Nor: normal rats, Micr: hereditary microphthalmic rats. DA: dopamine, NA: noradrenaline, A: adrenaline, 5-HT: serotonin.

thalamus. Neuronal processes were also well stained in the hypothalamus.

The concentration of monoamines in the substantia nigra and hypothalamus of normal and microphthalmic rats is shown in Fig. 2. In both normal and microphthalmic rats, the level of dopamine was about 2 -fold higher in the substantia nigra than that in the hypothalamus, but the level of noradrenaline was several times greater in the hypothalamus than in the substantia nigra. The levels of adrenaline in both groups of animals were depressed in the substantia nigra and hypothalamus. These patterns of monoamine concentrations in both the brain sites of the mutant were almost consistent with those of normal rats. In normal rats, the levels of serotonin in the substantia nigra and of dopamine in the hypothalamus were significantly higher in the dark (23:00) than in the light (11:00) period, but in the mutant did not extensively fluctuate. In hereditary microphthalmic rats, a circadian fluctuation in the catecholamine or serotonin level was not observed between light and dark periods. Thus, the metabolic aspects of monoamines in the substantia nigra and hypothalamus of hereditary microphthalmic rats did not differ from that of normal rats, although the diurnal fluctuation of hypothalamic dopamine level were differed between both rats.

In this study, the immunohistochemical analysis revealed that tyrosine hydroxylase was present in neuronal cell bodies in both the brain sites of hereditary microphthalmic rats as well as normal rats. Reuss et al. [8] have reported an immunohistochemical analysis in which tyrosine hyroxylase was present in neuronal 
perikarya and processes in the anteroventral periventricular subnucleus of the medial preoptic area, while dopamine-beta-hydroxylase was only seen in fibers and terminals. In the present experiment, the perikarya-like immunoreactive structures were also mainly observed in the substantia nigra and hypothalamus of normal and microphthalmic rats.

Although the patterns of the monoamine levels in the substantia nigra and hypothalamus of microphthalmic rats did not extensively differ from those of normal rats, the diurnal changes of dopamine level in the hypothalamus and of serotonin level in the substantia nigra observed in the normal rats, disappeared in the hereditary microphthalmic rats. Diurnal variations in brain monoamine levels have been the subject of many animal experiments [7, 8]. Simon et al. [12] have found that the diurnal variation of the dopamine level in the cortex, striatum, septum, amygdala, pons, and midbrain of rats shows ultradian rhythms. In contrast, Bobillier and Mouret [1] have reported a night-time increase of dopamine level in the brain anterior part of normal rats, while Reuss et al. [8] found that dopamine and 3,4dihydroxyphenyl acetic acid (DOPAC) concentrations in the medial preoptic area of the female rat hypothalamus decreased from day to night, but noradrenaline was doubled. We measured the concentrations of monoamines in the substantia nigra and hypothalamus of the normal rat in the middle of the light (11:00) and dark (23:00) periods, so that it was difficult to know whether the diurnal variation of dopamine exhibited was an ultradian or circadian rhythm. More sampling points will be needed to clarify the mechanism involved in the diurnal changes in the substantia nigra and hypothalamus of the normal rat.

We have previously reported that the hereditary microphthalmic rat exhibits circadian rhythms unentrained to the light cycle, i.e., a free-running circadian rhythm for spontaneous locomotor activity under a 12-hr LD cycle and free access to food condition [14]. We have also proposed that each animal maintains its circadian rhythm in the enzyme activities of pineal serotonin Nacetyltransferase [10] and hepatic and renal ornithine decarboxylase [11], which persists with various freerunning times, but the group rhythm disappears because it becomes desynchronized among animals.

\section{Acknowledgments}

We thank Mr. T. Tachibana for his technical assistance. This study was supported by research funds from Chosun University, Korea (1998-1999) and in part by a Grant-in-Aid for Scientific Research from the Ministry of Education, Science, and Culture of Japan (09460059).

\section{References}

1. Bobillier, P. and Mouret, J.P. 1971. Intern. J. Neuroscience 2: 271-282.

2. Browman, L.G. 1943. J. Comp. Physiol. Psychol. 36: 3346.

3. Ibuka, N. 1987. Physiol. Behav. 39: 321-326.

4. Ikeda, Y. and Chiba, Y. 1981. Zool. Mag. 90: 362-367.

5. Keller, R., Oke, A., Mefford, I., and Adams, R.N. 1976. Life Sciences 19: 995-1003.

6. Kobayashi, K. and Otani, K. 1981. J. Morphol. 167: 265276.

7. Mansbardt, J. and Wurtman, R.J. 1968. Nature 217: 574575.

8. Reuss, S., Hermes, B., Fuchs, E., and Hiemke, C. 1999. Neurosci. Lett. 266: 29-32.

9. Scheuch, G.C., Johnson, W., Conner, R.L., and Silver, J. 1982. J. Comp. Physiol. 149: 333-338.

10. Shim, S., Fu, Z., Kato, H., and Tanaka, H. 1997. Biosci. Biotechnol. Biochem. 61: 2113-2115.

11. Shim, S., Sugita, S., Sugahara, K., and Tanaka, H. 1997. Physiol. Behav. 62: 1365-1369.

12. Simon, M.L. and George, R. 1975. Neuroendocri. 17: 125138.

13. Sugita, S., Minematsu, S., Nagai, K., and Sugahara, K. 1996. Exp. Anim. 45: 115-124.

14. Tanaka, H., Shim, S., Hitomi, Y., Sugita, S., and Sugahara, K. 1999. Physiol. Behav. 67: 167-172.

15. Tokunaga, A., Sugita, S., Nagai, K., Tsutsui, K., and Ohsawa, K. 1997. Neurosci. Res. 27: 57-63. 